Preprint JINR E2-97-63

\title{
Dimensional reduction and BRST approach to the description of a Regge trajectory
}

\author{
A. Pashnev \\ and M. Tsulaial \\ JINR-Bogoliubov Laboratory of Theoretical Physics, \\ 141980 Dubna, Moscow Region, Russia
}

\begin{abstract}
The local free field theory for Regge trajectory is described in the framework of the BRST - quantization method. The corresponding BRST - charge is constructed with the help of the method of dimensional reduction.
\end{abstract}

Submitted to Modern Physics Letters A

\footnotetext{
*E-mail: pashnev@thsun1.jinr.dubna.su

${ }^{\dagger}$ E-mail: tsulaia@thsun1.jinr.dubna.su
} 


\section{Introduction}

The main problem in the description of the higher spin particles is removing of unphysical degrees of freedom from the theory. As was shown in [1], the corresponding lagrangian must have some invariance, which generalizes the gauge invariance of the electromagnetic field not only in the free case, but for interacting fields, as well.

On the free level such lagrangians was constructed both for massive and for massless particles of any spin as well as for massless supermultiplets [2]-[10]. In some sense the massless case is simpler and, hence, more investigated than the massive one. The hope is, that with the help of some Higgs-type effect some of the interacting particles acquire nonzero values of the mass.

In general the lagrangians for higher spins include additional fields. Some of them are auxiliary, others can be gauged away. The main role of these fields is to ensure, that only physical degrees of freedom are propagating ones. It means, that the basic field describes irreducible representation of the Poincare group - it is traceless, transverse and satisfies mass shell equation.

Naturally higher spin particles arise after quantization of classical extended objects such as string, relativistic oscillator [11]- [12], discrete string [13] etc. Physically they correspond to exited levels of the system and belong to Regge trajectories, each including infinite sequence of states with a spin linearly depending on the square of the mass. There are infinite number of Regge trajectories in the string and relativistic oscillator models and only one such trajectory in the discrete string model due to existing of additional second-class constraints. Such subdivision of all higher spin particles on Regge trajectories leads to the consideration of these Regge trajectories as independent objects for which it would be interesting to construct the lagrangian description.

One of the most economic and straightforward method of construction of lagrangians for systems with constraints is the method using BRST-charge of the corresponding firstly quantized theory. With the help of BRST-charge the lagrangians for the free field theory for particular values of spins [16] and for infinite tower of massless higher spin particles were constructed [15]. The analogous consideration for the massive fields is hampered by the presence of the second class constraints. The methods of converting them into the first class constraints with the help of additional variables were discussed in [17]-18]. Nevertheless, the strightforward application of this approach to the massive higher spins leads to the non-local lagrangian [19].

In the second part of the paper we describe the auxiliary Fock space and system of constraints in it which follow from the consideration of some classical models of extended objects.

In the third part of the paper we describe the massless case of [15] in $D+1$ dimensions which is the starting point for the consequent consideration. After that we fulfil the dimensional reduction from $D+1$ dimensional to $D$ dimensional space time. Formally it looks like some unitary transformation. As a result, the correct local free field lagrangian for Regge trajectory together with its daughter trajectories 
is constructed.

In the fourth part of the paper we write out as examples the lagrangians for lower spins $S=0,1,2$.

\section{Constraints in auxiliary space}

To describe all higher spins simultaneously it is convenient to introduce auxiliary Fock space generated by creation and annihilation operators $a_{\mu}^{+}, a_{\mu}$ with vector Lor entz index $\mu=0,1,2, \ldots D-1$, satisfying the following commutation relations

$$
\left[a_{\mu}, a_{\nu}^{+}\right]=-g_{\mu \nu}, g_{\mu \nu}=\operatorname{diag}(1,-1,-1, \ldots,-1) .
$$

In addition the operators $a_{\mu}^{+}, a_{\mu}$ can have some internal indices leading to more complicated spectrum of physical states. For simplicity we consider in this paper $a_{\mu}^{+}, a_{\mu}$ without additional indices.

The general state of the Fock space

$$
|\Phi\rangle=\sum \Phi_{\mu_{1} \mu_{2} \cdots \mu_{n}}^{(n)}(x) a_{\mu_{1}}^{+} a_{\mu_{2}}^{+} \cdots a_{\mu_{n}}^{+}|0\rangle
$$

depends on space-time coordinates $x_{\mu}$ and its components $\Phi_{\mu_{1} \mu_{2} \cdots \mu_{n}}^{(n)}(x)$ are tensor fields of rank $n$ in the space-time of arbitrary dimension $D$. The norm of states in this Fock space is not positively definite due to the minus sign in the commutation relation (2.1) for time components of creation and annihilation operators. It means that physical states must satisfy some constraints to have positive norm. These constraints arise naturally in the considerations of classical composite systems [12][13]. The corresponding quantum operators

$$
\begin{aligned}
& L_{0}=-p_{\mu}^{2}-\alpha^{\prime} a_{\mu}^{+} a_{\mu}, \quad L_{1}=p_{\mu} a_{\mu}, \quad L_{-1}=p_{\mu} a_{\mu}^{+}=L_{1}^{+}, \\
& L_{2}=\frac{1}{2} a_{\mu} a_{\mu}, \quad L_{-2}=\frac{1}{2} a_{\mu}^{+} a_{\mu}^{+}=L_{2}^{+}
\end{aligned}
$$

form the algebra

$$
\begin{gathered}
{\left[L_{0}, L_{ \pm 1}\right]=\mp \alpha^{\prime} L_{ \pm 1} \quad\left[L_{0}, L_{ \pm 2}\right]=\mp 2 \alpha^{\prime} L_{ \pm 2}} \\
{\left[L_{1}, L_{-2}\right]=-L_{-1} \quad\left[L_{-1}, L_{2}\right]=L_{1}} \\
{\left[L_{1}, L_{-1}\right]=-p_{\mu}{ }^{2}\left[L_{2} \cdot L_{-2}\right]=-a_{\mu}^{+} a_{\mu}+\frac{D}{2} \equiv G_{0}}
\end{gathered}
$$

The operators $L_{0}, L_{1}, L_{2}$ correspond to the mass shell, transversality and tracelessness conditions on the wavefunctions. The operators $L_{1}, L_{-1}$ and $L_{2}, L_{-2}$ are of 
second class and in general this system of constraints describes single Regge trajectory [12]-14.

There exist some different possibilities in consideration of general system of constraints (2.3)-(2.4). The truncated system $L_{0}, L_{ \pm 1}$ describes Regge trajectory together with its daughter trajectories. The operators $L_{ \pm 1}$ in this system are of second class as before. We describe the BRST - quantization of this system in the third part of the article.

The limit $\alpha^{\prime}=0$ of the system (2.3) corresponds to the massless infinite tower of spins with a single state at each value of the spin. In this case only the operators $L_{ \pm 2}$ are of second class. The BRST - quantization of this system as well as general system (2.3)-(2.4) will be given elsewhere.

The simplest system of first-class constraints

$$
\tilde{L}_{0}=-p_{\mu}{ }^{2}, L_{ \pm 1}
$$

corresponds to the massless tower of spins infinitely degenerated at each value of spin. The BRST - construction for this system of constraints was described in [15]- [16].

\section{Massless case and dimensional reduction}

In this section we consider the system with constraints

$$
L_{0}=-p_{\mu}{ }^{2}-\alpha^{\prime} a_{\mu}^{+} a_{\mu}+\alpha_{0}, \quad L_{1}=p_{\mu} a_{\mu}, \quad L_{-1}=p_{\mu} a_{\mu}^{+},
$$

where parameter $\alpha_{0}$ plays the role of intercept for Regge trajectory. In some sense this system is intermediate between the systems in [15] and [14] because it describes the Regge trajectory together with all daughter trajectories (due to the absence of the constraints $\left.L_{ \pm 2}\right)$. The commutation relation $\left[L_{1}, L_{-1}\right]=-p_{\mu}{ }^{2}$ means that $L_{ \pm 1}$ are the second class constraints . To construct the BRST - charge we can try to convert them into the first class constraints following the prescription of [17] - [18]. We introduce new operators $b$ and $b^{+}$with the commutation relations $\left[b, b^{+}\right]=1$ and modify the constraints to the following expressions:

$$
\begin{gathered}
\tilde{L}_{0}=-p_{\mu}{ }^{2}-\alpha^{\prime} a_{\mu}^{+} a_{\mu}+\alpha_{0}+\alpha^{\prime} b^{+} b \\
\tilde{L}_{-1}=L_{-1}+\sqrt{p_{\mu}^{2}} b^{+} \\
\tilde{L}_{1}=L_{1}+\sqrt{p_{\mu}^{2}} b \\
{\left[\tilde{L}_{1}, \tilde{L}_{-1}\right]=0, \quad\left[\tilde{L}_{0}, \tilde{L}_{1}\right]=-\alpha^{\prime} \tilde{L}_{1}, \quad\left[\tilde{L}_{0}, \tilde{L}_{-1}\right]=\alpha^{\prime} \tilde{L}_{-1} .}
\end{gathered}
$$

All of the modified constraints are of first class and BRST - charge construction is straightforward [19]. Nevertheless, the corrresponding lagrangian is not satisfactory one. Indeed, it is non-local due to presence of $\sqrt{p_{\mu}{ }^{2}}$ in the definitions 
of constraints $\tilde{L}_{ \pm 1}$. The natural way out of this difficulty is to replace $\sqrt{p_{\mu}^{2}}$ by $\sqrt{-\alpha^{\prime} a_{\mu}^{+} a_{\mu}+\alpha_{0}+\alpha^{\prime} b^{+} b}$, having new system of constraints with rather nontrivial commutation relations due to such complicated dependence from creation and annihilation operators.

The simplest way to construct BRST - charge with corresponding constraints is as follows. Firstly we consider massless case in $D+1$ dimensions with constraints

$$
\begin{aligned}
L_{0} & =-p_{\mu}^{2}+p_{D}^{2}, \quad \mu=0,1, \ldots, D-1, \\
L_{1} & =p_{\mu} a_{\mu}-p_{D} a_{D}, \\
L_{-1} & =p_{\mu} a_{\mu}^{+}-p_{D} a_{D}^{+} .
\end{aligned}
$$

Following to the standard procedure we introduce additional set of anticommuting variables $\eta_{0}, \eta_{1}, \eta_{1}^{+}$having ghost number one and corresponding momenta $\mathcal{P}_{0}, \mathcal{P}_{1}^{+}, \mathcal{P}_{1}$ with commutation relations:

$$
\left\{\eta_{0}, \mathcal{P}_{0}\right\}=\left\{\eta_{1}, \mathcal{P}_{1}^{+}\right\}=\left\{\eta_{1}^{+}, \mathcal{P}_{1}\right\}=1
$$

The nilpotent BRST - charge has the following form:

$$
Q=\eta_{0} L_{0}+\eta_{1}^{+} L_{1}+\eta_{1} L_{1}^{+}+\eta_{1} \eta_{1}^{+} \mathcal{P}_{0}
$$

Consider the total Fock space generated by creation operators $a_{\mu}^{+}, a_{D}^{+}, \eta_{1}^{+}, \mathcal{P}_{1}^{+}$. In addition each vector of the Fock space depends linearly on the real grassmann variable $\eta_{0}\left(\mathcal{P}_{0}\right.$ considered as corresponding derivative $\left.\mathcal{P}_{0}=\partial / \partial \eta_{0}\right)$

$$
|\chi\rangle=\left|\chi_{1}\right\rangle+\eta_{0}\left|\chi_{2}\right\rangle
$$

Ghost numbers of $\left|\chi_{1}\right\rangle$ and $\left|\chi_{2}\right\rangle$ are different if the state $|\chi\rangle$ has some definite one.

The BRST - invariant lagrangian in such Fock space can be written as

$$
L=-\int d \eta_{0}\langle\chi|Q| \chi\rangle
$$

To be physical, lagrangian $L$ must have zero ghost number. It means that vectors $|\chi\rangle$ and $\left|\chi_{1}\right\rangle$ have zero ghost numbers as well. In this case the ghost number of $\left|\chi_{2}\right\rangle$ is minus one. The most general expressions for such vectors are

$$
\begin{aligned}
\left|\chi_{1}\right\rangle & =\left|S_{1}\right\rangle+\eta_{1}^{+} \mathcal{P}_{1}^{+}\left|S_{2}\right\rangle, \\
\left|\chi_{2}\right\rangle & =\mathcal{P}_{1}^{+}\left|S_{3}\right\rangle,
\end{aligned}
$$

with vectors $\left|S_{i}\right\rangle$ having ghost number zero and depending only on bosonic creation operators $a_{\mu}^{+}, a_{D}^{+}$

$$
\left|S_{i}\right\rangle=\sum \phi_{\mu_{1}, \mu_{2}, \ldots \mu_{n}}^{n}(x) a_{\mu_{1}}^{+} a_{\mu_{2}}^{+} \ldots a_{\mu_{n}}^{+}\left(a_{D}^{+}\right)^{n}|0\rangle .
$$


Integration over the $\eta_{0}$ gives the following expression for the lagrangian in terms of $\left|S_{i}\right\rangle$

$$
\begin{aligned}
L= & \left\langle S_{1}\left|p_{\mu}{ }^{2}\right| S_{1}\right\rangle-\left\langle S_{2}\left|p_{\mu}{ }^{2}\right| S_{2}\right\rangle-\left\langle S_{3}|| S_{3}\right\rangle+ \\
& \left\langle S_{1}\left|L_{1}^{+}\right| S_{3}\right\rangle+\left\langle S_{3}\left|L_{1}\right| S_{1}\right\rangle-\left\langle S_{2}\left|L_{1}\right| S_{3}\right\rangle-\left\langle S_{3}\left|L_{1}^{+}\right| S_{2}\right\rangle .
\end{aligned}
$$

The nilpotency of the BRST - charge leads to the invariance of the lagrangian (3.12) under the following transformations

$$
\delta|\chi\rangle=Q|\Lambda\rangle
$$

The parameter of transformation must have ghost number -1 and can be written as $|\Lambda\rangle=\mathcal{P}_{1}^{+}|\lambda\rangle$, where $|\lambda\rangle$ belong to the Fock space generated by $a_{\mu}^{+}, a_{D}^{+}$and depends from the space - time coordinates.

Using together this invariance and equations of motion for the fields $\left|S_{i}\right\rangle$ one can show, that the lagrangian (3.27) describes the infinite number of higher spin massless particles with infinite multiplicity at each value of spin [15].

To obtain the description of the Regge trajectory in $D$ dimensions one can make use of the dimensional reduction procedure to the $D+1$ dimensional massless lagrangian. The masslessness condition in $D+1$ dimensions is

$$
p_{\mu}^{2}-p_{D}^{2}=0
$$

After the fixing $p_{D}=m$ with some arbitrary parameter $m$ this equation describes massive particle. In principle $m$ can depend from the spin of particle, leading to the Regge trajectory.

To describe the linear Regge trajectory we fix the following $x_{D}$ dependence of the Fock space vector $|\chi\rangle$ in (3.12):

$$
|\chi\rangle=\exp \left(i x_{D} \sqrt{\alpha^{\prime}\left(-a_{\mu}^{+} a_{\mu}+a_{D}^{+} a_{D}+\eta_{1}^{+} \mathcal{P}_{1}+\mathcal{P}_{1}^{+} \eta_{1}\right)+\alpha_{0}}\left|\chi^{\prime}\right\rangle \equiv U\left|\chi^{\prime}\right\rangle\right.
$$

The result of the substitution of (3.19) in the expression (3.12) is

$$
L=-\int d \eta_{0}\left\langle\chi^{\prime}|\tilde{Q}| \chi^{\prime}\right\rangle
$$

Where

$$
\begin{aligned}
\tilde{Q}= & \eta_{0}\left(\tilde{L}_{0}+\alpha^{\prime}\left(\eta_{1}^{+} \mathcal{P}_{1}+\mathcal{P}_{1}^{+} \eta_{1}\right)\right)+\eta_{1}^{+}\left(\tilde{L}_{1}+\mathcal{P}_{1}^{+} X \eta_{1} a_{D}\right)+ \\
& +\left(\tilde{L}_{1}^{+}+a_{D}^{+} \eta_{1}^{+} X \mathcal{P}_{1}\right) \eta_{1}+\eta_{1} \eta_{1}^{+} \mathcal{P}_{0}
\end{aligned}
$$

with the following notations used

$$
\begin{gathered}
\tilde{L}_{0}=-p_{\mu}{ }^{2}+\alpha^{\prime}\left(-a_{\mu}^{+} a_{\mu}+a_{D}^{+} a_{D}\right)+\alpha_{0} \\
\tilde{L}_{1}=p_{\mu} a_{\mu}-\sqrt{\alpha^{\prime}\left(-a_{\mu}^{+} a_{\mu}+a_{D}^{+} a_{D}\right)+\alpha^{\prime}+\alpha_{0}} a_{D}
\end{gathered}
$$




$$
\begin{gathered}
\tilde{L}_{1}^{+}=p_{\mu} a_{\mu}^{+}-a_{D}^{+} \sqrt{\alpha^{\prime}\left(-a_{\mu}^{+} a_{\mu}+a_{D}^{+} a_{D}\right)+\alpha^{\prime}+\alpha_{0}} \\
X=-\sqrt{\alpha^{\prime}}\left(\sqrt{-a_{\mu}^{+} a_{\mu}+a_{D}^{+} a_{D}+\frac{\alpha_{0}}{\alpha^{\prime}}+2}-\sqrt{-a_{\mu}^{+} a_{\mu}+a_{D}^{+} a_{D}+\frac{\alpha_{0}}{\alpha^{\prime}}+1}\right)
\end{gathered}
$$

The commutator of constraints (3.23) and (3.24) has rather complicated form:

$$
\left[\tilde{L}_{1}, \tilde{L}_{1}^{+}\right]=\tilde{L}_{0}+\alpha^{\prime}+a_{D}^{+} X \tilde{L}_{1}+\tilde{L}_{1}^{+} X a_{D}+a_{D}^{+} X^{2} a_{D}
$$

The new BRST - charge $\tilde{Q}(3.21)$ is nilpotent due to unitarity of the transformation $\tilde{Q}=U^{-1} Q U$. Our choice of square root dependence in the exponent in (3.19) leads, as we will see later, to the linear Regge trajectory. Replacement of this square root by any other function consistently gives Regge trajectory with more complicated dependence between spin and mass. The nilpotency of the BRST - charge evidently does not depend from the choice of this function.

In terms of $\left|S_{i}\right\rangle$ the lagrangian (3.20) has the folloqing form:

$$
\begin{aligned}
L= & -\left\langle S_{1}\left|\tilde{L}_{0}\right| S_{1}\right\rangle+\left\langle S_{2}\left|\left(\tilde{L}_{0}+2 \alpha^{\prime}\right)\right| S_{2}\right\rangle+\left\langle S_{1}\left|\tilde{L}_{1}^{+}\right| S_{3}\right\rangle+\left\langle S_{3}\left|\tilde{L}_{1}\right| S_{1}\right\rangle- \\
& \left\langle S_{2}\left|\left(\tilde{L}_{1}+X a_{D}\right)\right| S_{3}\right\rangle-\left\langle S_{3}\left|\left(\tilde{L}_{1}^{+}+a_{D}^{+} X\right)\right| S_{2}\right\rangle-\left\langle S_{3}|| S_{3}\right\rangle .
\end{aligned}
$$

The correswponding equations of motion are:

$$
\begin{aligned}
& \tilde{L}_{0}\left|S_{1}\right\rangle-\tilde{L}_{1}^{+}\left|S_{3}\right\rangle=0, \\
& \left(\tilde{L}_{0}+2 \alpha^{\prime}\right)\left|S_{2}\right\rangle-\left(\tilde{L}_{1}+X a_{D}\right)\left|S_{3}\right\rangle=0, \\
& \left|S_{3}\right\rangle-\tilde{L}_{1}\left|S_{1}\right\rangle+\left(\tilde{L}_{1}^{+}+a_{D}^{+} X\right)\left|S_{2}\right\rangle=0 .
\end{aligned}
$$

The transformation law $\delta\left|\chi^{\prime}\right\rangle=\tilde{Q}|\Lambda\rangle$ has the following component form:

$$
\begin{aligned}
\delta\left|S_{1}\right\rangle & =\tilde{L}_{1}^{+}|\lambda\rangle \\
\delta\left|S_{2}\right\rangle & =\left(\tilde{L}_{1}+X a_{D}\right)|\lambda\rangle \\
\delta\left|S_{3}\right\rangle & =\left(\tilde{L}_{0}+\alpha^{\prime}\right)|\lambda\rangle .
\end{aligned}
$$

One can show that using together (3.31) and equations of motion for the fields $\left|S_{i}\right\rangle$ one can eliminate the fields $\left|S_{2}\right\rangle$ and $\left|S_{3}\right\rangle$. Firstly we solve the equation

$$
\left|S_{3}\right\rangle+\left(\tilde{L}_{0}+\alpha^{\prime}\right)|\lambda\rangle=0
$$

using decompositions

$$
\left|S_{i}\right\rangle=\sum\left(a_{D}^{+}\right)^{n}\left|S_{i n}\right\rangle, \quad|\lambda\rangle=\sum\left(a_{D}^{+}\right)^{n}\left|\lambda_{n}\right\rangle .
$$

The equation (3.34) does not fix parameter $|\lambda\rangle$ completely. There will be residual invariance with parameter $\left|\lambda^{\prime}\right\rangle$ under the condition

$$
\left(\tilde{L}_{0}+\alpha^{\prime}\right)\left|\lambda^{\prime}\right\rangle=0
$$


After the elimination of the field $\left|S_{2}\right\rangle$ with the help of the equation $\left|S_{2}\right\rangle+\left(\tilde{L}_{1}+\right.$ $\left.X a_{D}\right)\left|\lambda^{\prime}\right\rangle=0$, which is consistent with the equations (3.29) and (3.36), the new parameter $\left|\lambda^{\prime \prime}\right\rangle$ will satisfy two conditions $\left(\tilde{L}_{0}+\alpha^{\prime}\right)\left|\lambda^{\prime \prime}\right\rangle=\left(\tilde{L}_{1}+X a_{D}\right)\left|\lambda^{\prime \prime}\right\rangle=0$. With the help of this parameter all fields $\left|S_{1 n}\right\rangle$, except $\left|S_{10}\right\rangle$ can be eliminated as well. It means that only $\left|S_{10}\right\rangle$ under conditions

$$
L_{0}\left|S_{1}\right\rangle=0, \quad L_{1}\left|S_{1}\right\rangle=0
$$

is a physical field. The second condition kills negative norm states of the Fock space. The first one - the mass shell condition - fixes the linear dependence between masses and spins of physical states.

In general the Fock space vectors $|\chi\rangle$ and $\left|\chi^{\prime}\right\rangle$ have complex vawefunctions. In the massless case one can impose the following reality conditions consistent with the equations of motion and transformation law (3.17): the coefficients in $\left|S_{1}\right\rangle$ and $\left|S_{2}\right\rangle$ are real as opposite to the coefficients in $\left|S_{3}\right\rangle$ and $|\lambda\rangle$, which are purely imaginary. The transformation (3.19), due to its complexity destroys such reality conditions for the massive case.

\section{Examples}

The total lagrangian (3.27) describes all spins from zero to infinity. The maximal spin at the level $m^{2}=\alpha_{0}+n \alpha^{\prime}$ is $n$. Due to luck of the tracelessness constraint, there are also spins $n-2, n-4 \ldots$ on this level. The part of the lagrangian describing this level contains fields in $\left|S_{1}\right\rangle,\left|S_{2}\right\rangle$ and $\left|S_{3}\right\rangle$ with $n, n-2$ and $n-1$ total numbers

of creation operators $a_{\mu}^{+}$and $a_{D}^{+}$. In this chapter we describe three simplest cases $n=0,1,2$.

For $n=0$ the only field

$$
\left|S_{1}\right\rangle_{0}=A|0\rangle
$$

gives contribution to the lagrangian:

$$
L=-A \partial_{\mu}^{2} A-\alpha_{0} A^{2}
$$

Obviously it describes spinless particle with $m^{2}=\alpha_{0}$.

For $n=1$ corresponding fields are:

$$
\left|S_{1}\right\rangle_{1}=\left(A_{\mu} a_{\mu}^{+}+i A a_{D}^{+}\right)|0\rangle \quad \text { and } \quad\left|S_{3}\right\rangle_{0}=i C|0\rangle
$$

The lagrangian

$$
\begin{array}{r}
L=A_{\nu} \partial_{\mu}{ }^{2} A_{\nu}-A \partial_{\mu}{ }^{2} A+\left(\alpha^{\prime}+\alpha_{0}\right) A_{\alpha}{ }^{2}-\left(\alpha^{\prime}+\alpha_{0}\right) A^{2}- \\
C^{2}-2 \sqrt{\left(\alpha^{\prime}+\alpha_{0}\right)} C A+C \partial_{\mu} A_{\mu}-A_{\mu} \partial_{\mu} C
\end{array}
$$


is invariant under the following gauge transformations

$$
\begin{aligned}
\delta A_{\mu} & =\partial_{\mu} \lambda \\
\delta A & =-\sqrt{\alpha^{\prime}+\alpha_{0}} \lambda \\
\delta C & =\left(\partial_{\mu}{ }^{2}+\alpha^{\prime}+\alpha_{0}\right) \lambda
\end{aligned}
$$

leading on mass shell to the equations

$$
A=C=0, \partial_{\nu} A_{\nu}=\left(\partial_{\nu}^{2}+\alpha^{\prime}+\alpha_{0}\right) A_{\mu}=0 .
$$

The corresponding formulas for $n=2$ are:

$$
\begin{gathered}
\left|S_{1}\right\rangle_{2}=\left(A_{\mu \nu} a_{\mu}^{+} a_{\nu}^{+}+2 i A_{\mu} a_{\mu}^{+} a_{D}^{+}+A a_{D}^{+} a_{D}^{+}\right)|0\rangle \\
\left|S_{2}\right\rangle_{0}=B|0\rangle \\
\left|S_{3}\right\rangle_{1}=\left(i C_{\mu} a_{\mu}^{+}+C a_{D}^{+}\right)|0\rangle \\
L=-2 A_{\nu \rho} \partial_{\mu}{ }^{2} A_{\nu \rho}-2\left(2 \alpha^{\prime}+\alpha_{0}\right) A_{\nu \rho}{ }^{2}+4 A_{\nu} \partial_{\mu}{ }^{2} A_{\nu}+4\left(2 \alpha^{\prime}+\alpha_{0}\right) A_{\nu}{ }^{2}- \\
2 A \partial_{\mu}{ }^{2} A-2\left(2 \alpha^{\prime}+\alpha_{0}\right) A^{2}+B \partial_{\mu}{ }^{2} B+\left(2 \alpha^{\prime}+\alpha_{0}\right) B^{2}-C^{2}+C_{\nu}{ }^{2}- \\
2 C_{\nu} \partial_{\mu} A_{\nu \mu}+2 A_{\nu \mu} \partial_{\mu} C_{\nu}-2 C \partial_{\mu} A_{\mu}+2 A_{\mu} \partial_{\mu} C-4 \sqrt{2 \alpha^{\prime}+\alpha_{0}} C A+ \\
4 \sqrt{2 \alpha^{\prime}+\alpha_{0}} C_{\mu} A_{\mu}+B \partial_{\mu} C_{\mu}-C_{\mu} \partial_{\mu} B+2 \sqrt{2 \alpha^{\prime}+\alpha_{0}} B C
\end{gathered}
$$

The gauge transformations with the parameter $|\lambda\rangle=\left(i \lambda_{\mu} a_{\mu}^{+}+\lambda a_{D}^{+}\right)|0\rangle$ give:

for $\left|S_{1}\right\rangle_{2}$ :

$$
\begin{aligned}
\delta A_{\mu \nu} & =\frac{1}{2}\left(\partial_{\mu} \lambda_{\nu}+\partial_{\nu} \lambda_{\mu}\right), \\
2 \delta A_{\mu} & =-\partial_{\mu} \lambda-\sqrt{2 \alpha^{\prime}+\alpha_{0}} \lambda_{\mu}, \\
\delta A & =-\sqrt{2 \alpha^{\prime}+\alpha_{0}} \lambda,
\end{aligned}
$$

for $\left|S_{2}\right\rangle_{0}$ :

$$
\delta B=-\partial_{\mu} \lambda_{\mu}-\sqrt{2 \alpha^{\prime}+\alpha_{0}} \lambda,
$$

and for $\left|S_{3}\right\rangle_{1}$ :

$$
\begin{aligned}
\delta C_{\mu} & =\left(\partial_{\nu}{ }^{2}+2 \alpha^{\prime}+\alpha_{0}\right) \lambda_{\mu}, \\
\delta C & =\left(\partial_{\nu}{ }^{2}+2 \alpha^{\prime}+\alpha_{0}\right) \lambda .
\end{aligned}
$$

After the gauge fixing the only nonvanishing field is $A_{\mu \nu}$ under conditions

$$
\left(\partial_{\rho}^{2}+2 \alpha^{\prime}+\alpha_{0}\right) A_{\mu \nu}=0, \partial_{\nu} A_{\mu \nu}=0 .
$$

Such equations describe simultaneously spin 2 and spin 0 particles. 


\section{Conclusions}

In this paper we have applied the BRST approach to the description of the free Regge trajectory. The corresponding BRST - charge was constructed with the help of dimensional reduction method. The resulting spectrum contains reducible representations of the Poincare group at each mass level due to luck of tracelessness constraint. It means that daughter trajectories also belong to the spectrum. The modification of the BRST approach to the single Regge trajectory will be given elsewhere.

Acknowledgments. One of us (A.P.) would like to thank M. Vasiliev and C. Preitschopf for useful discussions. This investigation has been supported in part by the Russian Foundation of Fundamental Research, grants 96-02-17634 and 9602-18126,grant MXL200, joint grant RFFR-DFG 96-02-00186G, and INTAS, grant 94-2317 and grant of the Dutch NWO organization.

\section{References}

[1] M.Fierz, W.Pauli. Proc.Roy.Soc., A173 (1939) 211

[2] E.S.Fradkin. JETP, 20 (1950) 27

[3] L.P.H.Singh, C.R.Hagen. Phys.Rev., D9 (1974) 898; Ibid D9 (1974) 910

[4] S.J.Chang. Phys.Rev., 161 (1967) 1308

[5] C.Fronsdal. Phys.Rev., D18 (1978) 3624

[6] J.Fang, C.Fronsdal. Phys.Rev., D18 (1978) 3630

[7] B.de Wit, D.Z. Freedman. Phys.Rev., D21 (1980) 358

[8] T. Curtright. Phys.Lett., B85 (1979) 219

[9] M.A. Vasiliev. Sov.J.Nucl.Phys., 32 (1980) 855 (439 in english translation)

[10] C. Aragone, S. Deser. Nucl.Phys.,B170 (1980) 329

[11] Y.S. Kim, Marilyn E. Noz. Phys.Rev., D12 (1975) 129;ibid D15 (1977) 335

[12] A.Barducci, D.Dominici. Nuovo Cim., A37 (1977) 385

[13] V.D.Gershun, A.I.Pashnev. Theor.Math.Phys., 73 (1987) 294

[14] A.I.Pashnev. Theor.Math.Phys., 78 (1989) 424 
[15] S.Ouvry, J.Stern., Phys.Lett., B177 (1986) 335

[16] Y. Meurice, Phys.Lett., B186 (1987) 189

[17] L.D. Faddeev, S.L. Shatashvili. Phys.Lett., B167 (1986) 225

[18] E.T. Egoryan, R.P. Manvelyan. Theor. Math.Phys., 94 (1993) 241

[19] A. Pashnev, M. Tsulaia., Preprint JINR E2-96-408, Dubna 1996;

hep-th/9611022 